\title{
Mapping the circumnuclear matter of NGC 1068 in X-rays
}

\author{
M. Guainazzi ${ }^{1}$ \\ Astrophysics Division, Space Science Department of ESA, ESTEC/SA, Postbus \\ 299, NL-2200 AG, The Netherlands \\ S. Molendi, P. Vignati \\ Istituto di Fisica Cosmica "G. Occhialini", C.N.R., Via Bassini 15, I-20133 \\ Milano, Italy \\ G. Matt \\ Dipartimento di Fisica, Università degli Studi "Roma Tre", Via della Vasca \\ Navale 84, I-00146 Roma, Italy \\ K. Iwasawa \\ Institute of Astronomy, University of Cambridge, Madingley Road, Cambridge \\ CB3 OHA, United Kingdom
}

\begin{abstract}
An-energy dependent variation of the X-ray emission from the archetypical "Comptonthick" Seyfert 2 galaxy NGC 1068 has been observed between two BeppoSAX observations, performed one year apart. This variation (at the $2.6 \sigma$ level) is highest in the 3-10 keV band and declines towards lower and higher energies. The most straightforward explanation is a variation of the primary nuclear continuum, which is scattered along our line of sight by an electron plasma. If this is indeed the case, this evidence allows us to obtain the first direct estimate of the location of the scattering medium, at $\sim 1$ pc from the nucleus.
\end{abstract}

Key words: galaxies: Seyfert - galaxies:individual: NGC 1068 - X-rays: general

1 Current address: XMM Science Operation Center, VILSPA, ESA, Apartado 50727, E-38080 Madrid, Spain. E-mail: mguainaz@xmm.vilspa.esa.es

Preprint submitted to Elsevier Preprint

1 April 2022 


\section{Introduction}

Compton-thick Seyfert 2 galaxies, i.e. sources in which the nucleus is obscured by matter with column densities exceeding $\sim \sigma_{t h}^{-1} \simeq 10^{24} \mathrm{~cm}^{-2}$, can be observed, in X-rays, only in scattered light. In the best known case, NGC 1068, at least two scattering components are known to be present (e.g. Iwasawa, Fabian \& Matt 1997; Matt et al. 1997). One originates from almost neutral material, possibly the inner surface of the molecular torus, envisaged by the Seyfert unification scenarios, Antonucci \& Miller 1985; Antonucci 1993. It is recognizable by the characteristic shape of the continuum, peaking between 20 and $30 \mathrm{keV}$, which is determined by the competition between photoabsorption and Compton scattering. A prominent iron line at $6.4 \mathrm{keV}$ comes along with it (George \& Fabian 1991; Matt, Perola \& Piro 1991). The other originates from ionized matter, and is a fainter replica of the nuclear radiation, at least as long as self-absorption effects are negligible. Lines from ionized elements, most commonly the $\mathrm{He}-$ and $\mathrm{H}$-like stages, are superposed to the latter.

Compton-thick Seyfert 2s are therefore ideally suited for studying the circumnuclear matter in AGN. From X-ray spectroscopy, much can be learned on the physical and chemical properties of the reflecting matter (e.g. Netzer 1996; Matt, Brandt \& Fabian 1996, Netzer \& Turner 1998). Even if a full exploitation of the line diagnostic capabilities should await high spectral resolution instruments, first results have already been obtained with moderate resolution detectors (Netzer \& Turner 1998; Guainazzi et al. 1999, G99 hereinafter). To map the location of the reflectors, instead, variability measurements are required. While an accurate mapping requires a good sampling performed with a high sensitivity detector (and even in this case a real reverberation technique cannot be applied due to the fact that the nuclear radiation is not directly observable), basic pieces of information can be derived by the crude comparison of observations made at different epochs, preferentially with the same instrument to avoid cross-calibration problems.

The Italian-Dutch X-ray satellite BeppoSAX (0.1-200 keV, Boella et al. 1997a) observed NGC 1068 twice, about one year apart. We therefore searched for variability between the two observations, and found an energy dependent one. In Sect. 2 a detailed description of the data reduction is presented, and possible systematic effects are discussed in Sect. 3. As the variability survived all our checks, in Sect. 4 we discuss the results and show that a possible explanation of the broadband X-ray variability is in term of a flattening of the nuclear component, mirrored by the ionized reflector. In Sect. 5 we derive some geometrical and physical properties of the warm scatterer (and of the other spectral components) implied by our results. 
Table 1

NGC 1068 BeppoSAX observation log

\begin{tabular}{|c|c|c|c|c|c|}
\hline ID & $\begin{array}{c}\text { Start Time } \\
\text { (UTC) }\end{array}$ & $\begin{array}{c}\text { End Time } \\
\text { (UTC) }\end{array}$ & $\begin{array}{c}\mathrm{T}_{\exp }^{\mathrm{LECS}} \\
(\mathrm{ks})\end{array}$ & $\begin{array}{c}\mathrm{T}_{\exp }^{\mathrm{MECS}} \\
(\mathrm{ks})\end{array}$ & $\begin{array}{l}\mathrm{T}_{\exp }^{\mathrm{PDS}} \\
(\mathrm{ks})\end{array}$ \\
\hline December 1996 & 30/12/1996 08:47:25 & 03/01/1997 05:27:50 & 61497 & 100150 & 62493 \\
\hline January 1998 & 11/01/1998 09:52:36 & 12/01/1998 08:07:50 & 15408 & 37331 & 17657 \\
\hline
\end{tabular}

\section{Observation and data reduction}

NGC 1068 was observed by BeppoSAX on December 1996 and January 1998 (see Tab. 1). Of the four co-aligned instruments, comprising the BeppoSAX scientific payload, we will deal in this paper with data from the Low Energy Concentrator Spectrometer (LECS, 0.1-10 keV, Parmar et al. 1997), the Medium Energy Concentrator Spectrometer (MECS, 1.8-10.5 keV, Boella et al 1997b) and the Phoswitch Detector System (PDS, 13-200 keV; Frontera et al. 1997). LECS and MECS are imaging gas proportional counters, which use identical concentrator systems, with moderate energy resolution $(\simeq 8 \%$ at $6 \mathrm{keV})$. The MECS effective area in the two units configuration discussed in this paper is about twice as the LECS one in the overlapping energy band. The PDS consists of four independent crystal units arranged in pairs, each mounted on a rocking collimator to achieve a continuous monitoring of the background with a duty-cycle of $96 \mathrm{~s}$.

Data reduction followed standard procedures, as described in G99. In particular, Good Time Intervals (GTI) for scientific products accumulation were defined according to the following selection criteria: $a$ ) the angle between the pointing directions and the Earth's limb was higher than $4^{\circ}$; b) passages through the South Atlantic Geomagnetic Anomaly were excluded; c) the angle between the pointing direction and the direction of the Sun was greater than $60^{\circ}$. Count rates were extracted from circular regions of radius 8' and 4' around the NGC 1068 centroid in the LECS and MECS, respectively. No other point source is detected in these areas at a level higher than $6 \times 10^{-14}$ and $1.6 \times 10^{-13} \mathrm{erg} \mathrm{cm}^{-2} \mathrm{~s}^{-1}$ (3 $\sigma$ local background fluctuations) in the $0.1-2 \mathrm{keV}$ and 2-10 keV energy bands, respectively. For comparison, G99 report average fluxes of 1.1 and $0.5 \times 10^{-11} \mathrm{erg} \mathrm{cm}^{-2} \mathrm{~s}^{-1}$ for the BeppoSAX observations of NGC 1068 in the same bands. PDS data were additionally screened by removing five minutes intervals after any SAGA passage, to allow gain recovery to nominal values after instrumental switch-offs.

We have performed an analysis of several BeppoSAX Crab observations to estimate if aging effects in the detectors could produce a secular variation of the observed count rate. No effect of this kind is observed, the upper limit 
Table 2

LECS background subtracted count rates (in units of $10^{-2} \mathrm{~s}^{-1}$ ) when the three different techniques of background subtraction after Parmar et al. (1999) are adopted (details in text)

\begin{tabular}{lccc}
\hline \hline & $0.1-1 \mathrm{keV}$ & $1-3 \mathrm{keV}$ & $3-10 \mathrm{keV}$ \\
\hline \multicolumn{2}{l}{ December 1996} & & \\
BLS & $6.29 \pm 0.10$ & $4.07 \pm 0.08$ & $1.51 \pm 0.07$ \\
FOV & $6.58 \pm 0.11$ & $4.02 \pm 0.09$ & $1.37 \pm 0.07$ \\
RM & $6.28 \pm 0.11$ & $4.00 \pm 0.09$ & $1.55 \pm 0.08$ \\
January 1998 & & \\
BLS & $5.82 \pm 0.20$ & $4.01 \pm 0.17$ & $1.60 \pm 0.13$ \\
FOV & $6.10 \pm 0.20$ & $3.96 \pm 0.18$ & $1.45 \pm 0.13$ \\
RM & $5.81 \pm 0.20$ & $3.94 \pm 0.18$ & $1.64 \pm 0.13$ \\
\hline \hline
\end{tabular}

being $0.3 \%$ and $0.7 \%$ for the LECS and MECS, respectively. The count rates obtained from the two NGC 1068 observations may be hence directly compared.

\section{$3 \quad$ LECS data reduction issues}

One of the most critical issues in estimating the variability of a relatively faint source as NGC 1068 is the background subtraction. In Tab. 2 we compare the count rates in three energy bands $(0.1-1 \mathrm{keV} ; 1-3 \mathrm{keV} ; 3-10 \mathrm{keV})$ when the three background subtraction techniques described in Parmar et al. (1999) are employed. They are: a) background extracted from blank sky deep exposures, accumulated by the BeppoSAX Science Data Center (SDC) in the first three years of operative life of the mission (BLS); b) background extracted from two semi-annuli in the LECS field of view, suitably renormalized to the expected counts under the source extraction region (FOV); c) cosmic background estimated from the 7-bands ROSAT/PSPC All Sky Survey source-removed count rates $(\mathrm{RM})$, and added to the instrumental background. The results differ by about $5 \%, 2 \%$ and $12 \%$ in the three bands, respectively. This differences might be due to local granularity in the contribution of the cosmic background, which is globally mapped on scales not lower than $2^{\circ}$ (Snowden et al. 1995). On the other hand, the instrumental LECS background is known to exhibit secular variations with a dynamical range of about a factor two (T.Oosterbroek, private communication). It is therefore impossible to tell which of the three techniques is giving the "true" result (see also the discussion in Parmar et al. 1999). Although the count rates uncertainties are dominated by system- 
Table 3

MECS background subtracted count rates (in units of $10^{-2} \mathrm{~s}^{-1}$ )

\begin{tabular}{lll}
\hline \hline $1.5-3.0 \mathrm{keV}$ & $3.0-5.0 \mathrm{keV}$ & $5.0-10.0 \mathrm{keV}$ \\
\hline December 1996 & \\
$2.40 \pm 0.05$ & $1.44 \pm 0.04$ & $2.36 \pm 0.05$ \\
January 1998 & \\
$2.39 \pm 0.08$ & $1.63 \pm 0.07$ & $2.57 \pm 0.09$ \\
\hline \hline
\end{tabular}

atic effects, the ratio between the count rates observed in the two BeppoSAX observations is independent of the background subtraction method used. Assuming the BLS one (the same adopted normally in spectral fitting), the ratio of the January 1998 versus the December 1996 count rates are: $0.93 \pm 0.04$, and $0.99 \pm 0.05$ and $1.06 \pm 0.11$ in the $0.1-1 \mathrm{keV}, 1-3 \mathrm{keV}$ and $3-10 \mathrm{keV}$ energy bands, respectively.

Count rate variations by a few percent could be ascribed to the a different positions of the source in the LECS field of view. It is in facts crossed by a mesh of fine grids, which cause a $\sim 5 \%$ modulation of the count rates on scales $\sim 10 "$, and by the strongback support rib, which causes a $\simeq 30 \%$ absorption at regular distances of 2' (along both the $\mathrm{X}$ and $\mathrm{Y}$ axis of the focal plane). The former effect has a much lower spatial scale than the PSF width $\left(\simeq 4^{\prime}\right.$ at $0.28 \mathrm{keV} ; \simeq 0.9^{\prime}$ at $\left.6 \mathrm{keV}\right)$, and is therefore averaged out when wide enough extraction radii are taken. The latter effect is unlikely to introduce a significant variability. The best-fit centroid of the source profiles differ by $\simeq 40$ " between the two BeppoSAX observations. On this scale, we estimate a count rate difference $\lesssim 1.5 \%$, well within the statistical uncertainties of our measures.

Response matrices appropriated for the source position in the two observations were extracted and used in the joint LECS/MECS spectral fitting. The results of the spectral fittings, described in detail in Appendix below, confirm the above outcomes.

\section{MECS data reduction issues}

In Tab. 4 we report the MECS background subtracted count rates for the two observations of NGC 1068 in the $1.5-3 \mathrm{keV}, 3-5 \mathrm{keV}$ and 5-10 keV energy bands. The softest ratio is consistent with unity, while for $\mathrm{E}>3 \mathrm{keV}$ the ratio is larger than 1 at the $2.6 \sigma$ level.

The change in the position of the source within the MECS detector from 
one pointing to the other (constrained by the better MECS statistics to be $\lesssim 30$ ") implied a difference in the vignetting $\lesssim 1 \%$. The gain is stable between the two observations. The difference between the best-fit centroids of the prominent iron line in the channel space between the two observations is $0.6 \pm 0.9$ PI ( 1 channel corresponds to $\sim 50 \mathrm{eV}$ ). We note that the MECS does not suffer of any support grid mesh obscuration problem. A support ring is present at about 10' from the center of the field of view and is almost concentric with it. No change of the MECS effective area on arc minute scales is therefore expected, other than that ascribed to the instrumental vignetting. Background subtraction has been performed by using blank field observations. To verify that the background during the two NGC 1068 observations is stable and consistent with that obtained from the blank fields, we have accumulated the count rates from the outer region of the detectors, where the contribution from the source is negligible. We find that the background count rates vary by less than $\sim 6 \%$ between the two observations. Considering that the background count rate contributes $\sim 8 \%$ to the source plus background count rate, we estimate that any spurious source count rate variation induced by inaccurate background subtraction will be smaller than $\sim 0.5 \%$. A systematic error of $1.5 \%$ will be hereinafter conservatively added to the MECS count rates to account for possible vignetting estimate and background subtraction inaccuracies.

\section{Comparison between the broadband spectra}

In Fig. 1 we show the ratio between the January 1998 and the December 1996 spectra. This plot includes also the ratio of the PDS count rates in the $13-30 \mathrm{keV}$ energy band. As noted before, the count rate ratio is $\sim 0.9$ below $1 \mathrm{keV}$, becomes consistent with unity in the 1-3 keV band, reaches a maximum (1.098 \pm 0.038$)$ between 3 and $10 \mathrm{keV}$ and then decreases again, returning consistent with 1 above $15 \mathrm{keV}$.

The maximum of the variability occurs in the energy range where the contribution of the warm scattered component is the highest (G99). The amount of the observed variability is admittedly small, and the spectral complexity hampers a clear identification of which component(s) is responsible for it. However, a comparison of Fig. 1 with G99 suggests that the observed variability might be due to a flattening of the warm scattered component around a pivot point located somewhere in the 1-4 keV band. More complex scenarios cannot be ruled out, and the interplay of spectral steepness and normalizations can further contribute to confuse the picture. Variability measurements of the lines associated with one or both the reflection components should help enlightening on the real behavior, but for this purpose much higher spectral resolution detectors are required. 
Fig. 1. Count rate ratio between the January 1998 and December 1996 observations as a function of energy. Empty squares: LECS; filled circles: MECS; cross: PDS. The dotted lines indicate the MECS measures in the $3-5 \mathrm{keV}$ and $5-10 \mathrm{keV}$ separately. The solid and dashed line in the PDS data point represent the statistical and statistical plus systematic uncertainties after Guainazzi \& Matteuzzi (1997). The $3-10 \mathrm{keV}$ LECS measure $(1.06 \pm 0.11)$ is consistent with the MECS one and not shown for sake of clarity

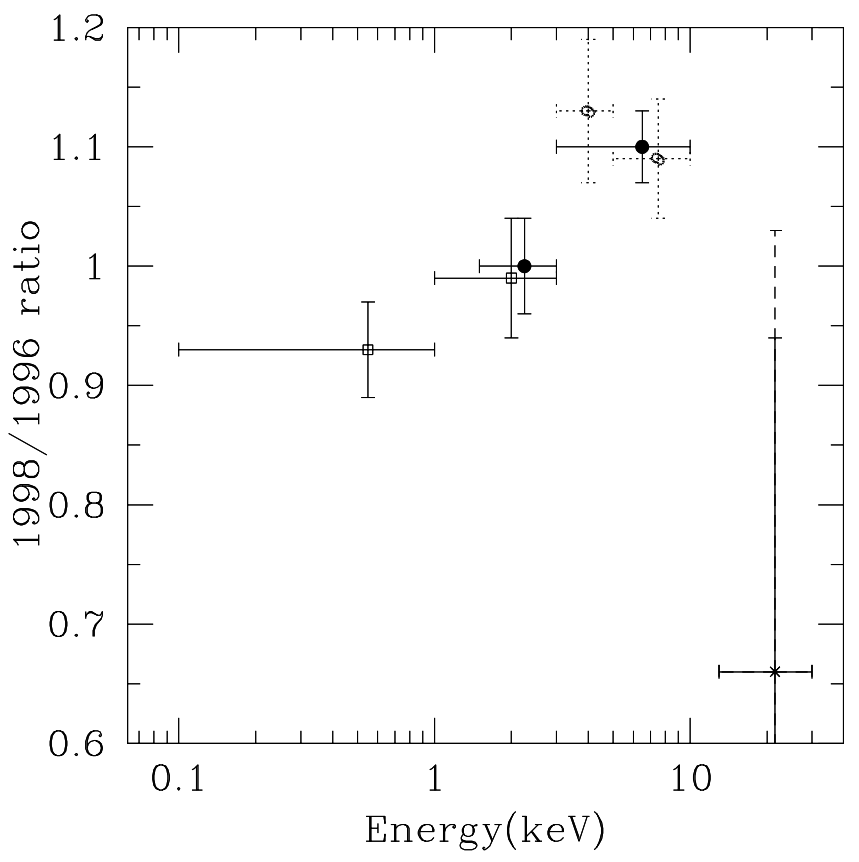

In Appendix, we show the comparison between the fitting results for the December 1996 and January 1998 spectra.

\section{Discussion}

We report on the observation of an energy dependent flux variability in NGC 1068 on timescales of about one year. The flux ratio of the 1998 over the 1996 observations is higher than 1 between 3 and $10 \mathrm{keV}$, and decreases towards lower and higher energies. Although the result is significant at the $2.6 \sigma$ level only, its possible implications are rather far-reaching, and we cannot refrain to discuss them in this Section. The next generation of high throughput, high resolution $\mathrm{X}$-ray detectors will allow a robust testing of the above results and of the forthcoming hypothesis.

General arguments on the spectral deconvolution suggest that the observed variation is best explained in terms of a change of the warm reflection component, echoing the same variation in the nuclear spectrum. The statistics 
is not good enough, however, to tell whether it is the spectral index or the normalization of the warm-scattered component (or both), that is responsible for the observed variability (see Appendix). Similarly, our data cannot test independently the hypothesis that self-absorbing effects in the warm-scattered component are negligible.

Apart from the details, it is rather plausible that the measured variation is mainly due to the warm reflection component. This may permit to derive information on the size and location of the reflecting matter. Light crossing arguments suggest that the size of the region cannot exceed one light-year, i.e one-third of a parsec. As the light-curve of the nuclear emission is unknown, we cannot determine with certainty the distance of the reflector to the nucleus. However, an order-of-magnitude estimate may be derived by recalling that the $2-10 \mathrm{keV}$ luminosity of the warm reflection component is about $1.5 \times 10^{41}$ $\operatorname{erg~s}{ }^{-1}$, and that its Thomson optical depth, $\tau_{T}$, is estimated to be a few $\times 10^{-3}$ (G99). The relation between nuclear and reflected luminosity may be written as:

$$
L_{r e f}=\tau_{T} f L_{n u c}
$$

where $f$ is a geometrical factor basically equal to the solid angle subtended by the reflecting matter to the nucleus, in units of $4 \pi$. Therefore, if the nuclear luminosity is not much larger than $10^{44} \mathrm{erg} \mathrm{s}^{-1}$ (Iwasawa et al. 1997), $f$ cannot be much lower than 1 . If this is the case, the distance of the reflecting region from the nucleus cannot be much larger than its size, and should therefore be of the order of a parsec or less. This crude estimate puts the warm scattering medium in an intermediate location between the Broad (Peterson et al. 1991; Stirpe et al. 1994; Dietrich et al. 1998) and the Narrow Line Regions (Axon et al. 1997). This supports the idea that this medium coincides with that responsible for the almost energy-independent scattering of the nuclear continuum, observed as the appearance of broad lines in the polarized light optical spectra of NGC 1068 (Antonucci \& Miller 1985). Because in this source the torus is probably observed almost edge-on (Greenhill et al. 1997; Matt et al. 1997), the warm reflector, in order to be visible, must be placed outside the torus itself. This implies a rather compact torus, with inner side of order of $1 \mathrm{pc}$ or less. This is in good agreement with the radio continuum measurements, which suggest the presence of a sub-parsec edge-on disk around the NGC 1068 nucleus (Gallimore et al. 1998).

Netzer \& Turner (1998) and G99 suggested that the bulk of the ionized emission lines observed in the soft X-ray spectrum of NGC 1068 originates in the same medium. The fact that transitions from He-like stages of elements from oxygen to iron are present, suggests that the ionization and/or geometrical structure of the scattering medium must be complex. This would be confirmed by the fact that, while most of the lines do not change significantly between the 
two observations, the $\mathrm{K}_{\alpha}$ Nexi varies by a factor two (see Appendix). Given the instrumental and statistical limits of our measurements, we refrain from pursuing this point any further. This results, however, indicates how profitable the study of the line dynamics could be, when the new generation of high resolution/high throughput detectors will be operative. New constraints on the physical conditions of the matter in the circumnuclear AGN environment can be set if monitoring with a sufficiently fine sampling will be available.

Comparatively little can be said on the variability of the other spectral components (see Appendix). The Compton-reflected continuum is detected only in the longer December 1996 observation. However, the January 1998 upper limit on its $5-200 \mathrm{keV}$ flux is not inconsistent with the December 1996 one. The thermal component parameters are remarkably constant. Again, this strengthens the idea that this component is connected to the strong starburst ring of approximately $1 \mathrm{kpc}$ size [i.e.: about 15" at the distance of $14.4 \mathrm{Mpc}$ (Tully 1988)], which protrudes a bar towards the nucleus (Scoville 1988). Actually, half of the emission at $0.8 \mathrm{keV}$ comes from an extended region of $\simeq 13 \mathrm{kpc}$ scale (Wilson et al. 1992). It is therefore straightforward to think that the BeppoSAX soft X-ray spectrum is dominated by the same component.

\section{APPENDIX - Time-resolved spectroscopy: an exercise}

We have tried to separately fit the best model of G99 to the December 1996 and January 1998 LECS/MECS/PDS spectra. In principle this procedure could allow us to determine exactly which spectral component(s) is responsible for the observed variability. In practise this is a very hard task, although BeppoSAX at least has the advantage, over previous missions, of the broad energy coverage which allows the best modeling of the continuum so far. We report the relevant results hereinafter mainly for sake of completeness. We extracted the MECS spectra from a region of 6' to achieve a better signal-to-noise ratio. The continuum model is characterized by the spectral index and normalization of the warm scattered $\left(\Gamma_{\mathrm{ws}}, \mathrm{N}_{\mathrm{ws}}\right)$ and Compton-reflected $\left(\Gamma_{\mathrm{cr}}, \mathrm{N}_{\mathrm{cr}}\right)$ primary continuum components, and by the temperature, abundance and normalization of the thermal component $\left(\mathrm{kT}, \mathrm{Z}, \mathrm{N}_{\mathrm{th}}\right.$; the mekal model in XsPEC has been used). The Galactic contribution to the photoelectric absorption has been held fixed to the value $2.9 \times 10^{20} \mathrm{~cm}^{-2}$, following Murphy et al. (1996), which is consistent with the value obtained in both the December 1996 and January 1998 fits if left as a free parameter. The results are summarized in Tab. 4 and Tab. 5 for the continuum and emission lines, respectively. Uncertainties are shown at $1 \sigma$ level for two interesting parameters $\left(\Delta \chi^{2}=2.30\right)$ for all the spectral components, except for the thermal optically thin plasma, for which the values corresponding to three interesting parameters are reported $\left(\Delta \chi^{2}=3.53\right)$. In the December 1996 observation, $\Gamma_{\mathrm{ws}}$ and $\Gamma_{\mathrm{cr}}$ turned out to be mutually consis- 
Table 4

Best-fit results with the baseline G99 model for the December 1996 and January 1998 BeppoSAX NGC 1068 observations

\begin{tabular}{|c|c|c|c|}
\hline Parameter & 1996 December & January & 1998 \\
\hline$\Gamma_{\mathrm{ws}}$ & $2.09 \pm 0.16$ & $1.26 \pm_{0.13}^{0.28}$ & $1.8 \pm 0.3$ \\
\hline $\mathrm{N}_{\mathrm{ws}}{ }^{a}\left(10^{-4}\right.$ photons $\left.\mathrm{s}^{-1} \mathrm{~cm}^{-2}\right)$ & $8.3 \pm_{0.7}^{1.0}$ & $5.0 \pm 1.6$ & $7 \pm_{3}^{4}$ \\
\hline$\Gamma_{\mathrm{cr}}$ & $\equiv \Gamma_{\mathrm{ws}}$ & $2.1^{b} / 1.25^{b}$ & $2.09^{b}$ \\
\hline $\mathrm{F}_{\mathrm{cr}}^{5-200 \mathrm{keV}}\left(10^{-11} \mathrm{erg} \mathrm{s}^{-1} \mathrm{~cm}^{-2}\right)$ & $3.2 \pm_{1.5}^{1.7}$ & $<1.7 /<2.9$ & $3.2^{b}$ \\
\hline $\mathrm{kT}(\mathrm{eV})$ & $390 \pm 40$ & $390 \pm_{60}^{50}$ & $380 \pm{ }_{80}^{60}$ \\
\hline $\mathrm{Z}(\%)$ & $1.6 \pm_{0.6}^{0.8}$ & $<1.3$ & $<1.9$ \\
\hline $\mathrm{N}_{\mathrm{th}}^{c}\left(10^{-2} \mathrm{~s}^{-5}\right)$ & $5.4 \pm 0.8$ & $5.7 \pm_{1.2}^{1.4}$ & $5.3 \pm_{1.3}^{1.5}$ \\
\hline$\chi^{2} / \operatorname{dof}$ & $365.6 / 333$ & $150.0 / 156$ & $156.2 / 156$ \\
\hline
\end{tabular}

${ }^{a}$ normalization at $1 \mathrm{keV}$ of the warm scattered component

${ }^{b}$ fixed

${ }^{c}$ normalization of the thermal component

tent, and therefore we report in Tab. 5 the results when these parameters are tied together. The only significant difference between the two fits is that the warm scattered component is much flatter and brighter in January 1998, the 2-10 keV flux increasing from $\left(1.84 \pm_{0.18}^{0.22}\right)$ to $\left(3.4 \pm_{1.1}^{0.7}\right) \times 10^{-12} \mathrm{erg} \mathrm{cm}^{-2} \mathrm{~s}^{-1}$. This suggest that it has pivoted around about $1 \mathrm{keV}$ between the two observations.

The Compton reflected component is actually detected only in the December 1996 observation, which has by far the higher signal-to-noise ratio, even if the upper limit of the $5-200 \mathrm{keV}$ flux in the second observation is consistent with the value in the first one (Table 5). The clear presence of the $6.4 \mathrm{keV}$ iron line also in the second observation (see below) strongly argues against the disappearing of the cold reflection. If the $\mathrm{CR}$ component is forced to be the same, in shape and flux, as in the first observation, it is actually impossible to tell whether it was the spectral index or the normalization of the WS component that changed (see column 4 of tab. 5).

In Tab. 4, we show the best-fit results for the emission lines, when the continuum models of columns 2 and 3 of Tab. 4 are employed. Most of them do not exhibit a variation of either the best-fit centroid energy or the intensity/Equivalent Width (EW). The only remarkable exception if the NexI one, whose flux doubles when the warm scattered continuum flattens, yielding an increase of the EW by a comparable factor. On the other hand, the ionized iron lines do not show any significant variation of the intensity, while the underlying warm scattered continuum increased by a factor about two. This would imply a decrease of their EW by the same factor, rather surprising if 
Table 5

Emission line best-fit parameters. The continuum models are those in columns 2 and 3 (for $\Gamma_{\text {cr }}=1.25$ ) in Tab. 4 for the December 1996 and January 1998 observations, respectively. Equivalent Width (EW) are calculated against the warm scattered continuum only, except the FeI, which is given against the Compton-reflected continuum only

\begin{tabular}{lccc}
\hline \hline Line & $\mathrm{E}$ & $\mathrm{I}$ & $\mathrm{EW}$ \\
& $(\mathrm{keV})$ & $\left(\right.$ photons cm $\left.{ }^{-2} \mathrm{~s}^{-1}\right)$ & $(\mathrm{eV})$ \\
\hline December 1996 & & \\
NeIX & $0.95 \pm_{0.03}^{0.02}$ & $\left(4.2 \pm_{1.2}^{1.1}\right) \times 10^{-4}$ & $570 \pm_{160}^{150}$ \\
SixiII & $1.88 \pm_{0.02}^{0.03}$ & $(8.6 \pm 1.3) \times 10^{-5}$ & $460 \pm 70$ \\
SXV & $2.46 \pm 0.04$ & $\left(3.1 \pm_{0.7}^{0.6}\right) \times 10^{-5}$ & $280 \pm_{60}^{50}$ \\
FeI & $6.4^{a}$ & $\left(5.3 \pm_{0.8}^{0.7}\right) \times 10^{-5}$ & $1400 \pm_{800}^{700}$ \\
FexXV & $6.7^{a}$ & $\left(5.3 \pm_{1.0}^{1.1}\right) \times 10^{-5}$ & $3500 \pm 800$ \\
FexXVI & $6.96^{a}$ & $\left(2.1 \pm_{0.7}^{0.9}\right) \times 10^{-5}$ & $1500 \pm_{500}^{600}$ \\
\hline January & 1998 & & \\
NeIX & $0.91 \pm_{0.02}^{0.04}$ & $\left(8 \pm_{2}^{3}\right) \times 10^{-4}$ & $1500 \pm_{400}^{600}$ \\
SixIII & $1.84 \pm 0.05$ & $\left(8 \pm_{2}^{3}\right) \times 10^{-5}$ & $380 \pm_{90}^{140}$ \\
SxV & $2.45 \pm_{0.05}^{0.06}$ & $\left(5.0 \pm_{1.3}^{1.4}\right) \times 10^{-5}$ & $330 \pm 90$ \\
FeI & $6.4^{a}$ & $\left(6.0 \pm_{1.5}^{1.4}\right) \times 10^{-5}$ & $>2200$ \\
FexXV & $6.7^{a}$ & $\left(5 \pm_{2}^{4}\right) \times 10^{-5}$ & $1400 \pm_{800}^{700}$ \\
FexXVI & $6.96^{a}$ & $\left(3.4 \pm_{1.6}^{1.5}\right) \times 10^{-5}$ & $1000 \pm_{600}^{400}$ \\
\hline \hline
\end{tabular}

the continuum and the lines are produced in one and the same medium. No problems of this kind are present with the fit of column 4 of Tab. 4 , however, which is therefore again to be preferred on plausibility grounds.

\section{ACKNOWLEDGMENTS}

BeppoSAX is a joint Italian-Dutch program. MG acknowledges the receipt of an ESA Research Fellowship. An anonymous referee is gratefully acknowledged for a careful scrutiny of the manuscript and several comments, which greatly improved the quality of the paper. 


\section{References}

[1] Antonucci R., 1993, ARA\&A, 31, 473

[2] Antonucci R., Miller J.S., 1985, ApJ, 297, 621

[3] Axon D.J., Marconi A., Capetti A., et al., 1998, ApJ, 496, L75

[4] Boella G., Butler R., Perola G.C., 1997a, A\&AS, 112, 299

[5] Boella G., Chiappetti L., Conti G., et al., 1997, A\&AS, 122, 327

[6] Dietrich M., Peterson B.M., Albrecht P., et al., 1998, ApJS, 115, 185

[7] Frontera F., Costa E., Dal Fiume D., et al., 1997, A\&AS, 122, 371

[8] Gallimore J.F., Baum S.A., O’Dea C.P., 1997, Nature, 388, 852

[9] George I.M., Fabian A.C., 1991, MNRAS, 249, 352

[10] Greenhill L.J., Ellingsen S.P., Norris R.P., et al., 1997, ApJ, 474, L103

[11] Guainazzi M., Matt G., Antonelli L.A., et al., 1999, MNRAS, in press (astroph/9905261)

[12] Guainazzi M., Matteuzzi A., 1997, SDC-TR-014

[13] Iwasawa K., Fabian A.C., Matt G., 1997, MNRAS, 289, 443

[14] Matt G., Brandt W.N., Fabian A.C., 1996, MNRAS, 280, 823

[15] Matt G., Guainazzi M., Frontera F., et al., 1997, A\&A, 325, L13

[16] Matt, G., Perola, G.C., Piro, L. 1991, A\&A, 247, 25

[17] Murphy E.M., Lockman F.J., Laor A., Elvis M., 1996, ApJSS, 105, 369

[18] Netzer H., 1996, ApJ, 473, 781

[19] Netzer H., Turner T.J., 1997, ApJ, 488, 694

[20] Parmar A.N., Martin D.D.E., Bavdaz M., et al., 1997, A\&AS, 122, 309

[21] Parmar A.N., Oosterbroek T., Orr A., et al., 1997, A\&AS 136, 407

[22] Peterson B.M., Balonek T.J., Barker E.S., et al., 1991, ApJ, 368, 199

[23] Scoville N.Z., 1988, ApJ, 327, 61

[24] Snowden S.L., Freyberg M.J., Plucinsky P.P., et al., 1995, ApJ, 454, 643

[25] Stripe G.M., Winge C., Altieri B., et al., 1994, ApJ, 425, 609

[26] Tully R.B., 1988, "Nearby Galaxy Catalog", Cambridge University Press

[27] Wilson A.S., Elvis M., Lawrence A., Bland-Hawthorn J., 1992, ApJ, 391, L75 